\title{
A survey of Radiative Coolers in the Literature
}

\author{
Jyotirmoy Mandal \\ University of California, Los Angeles \\ Correspondence: jyotirmoymandal@ucla.edu
}

\section{Preface}

This work is intended to be is a resource on prior radiative cooling designs in the literature, and will be periodically updated with new findings. The readers are requested to note that the resource reflects the literature as known by the author. As such, it cannot be used to determine novelty of new designs, but is useful for identifying precedents.

Some additional points to note:

- The emittances and reflectances quoted here are all measured using spectral data extracted from papers, rather than reported by authors. This is to ensure consistency in reported performances for the sake of comparison. Care has been taken to minimize errors during the data extraction process (which is estimated to be $<0.0025$, or $0.25 \%$ ).

- Solar reflectance of the data presented here has been measured relative to the AM1 spectrum. Most papers use AM 1.5, but as Levinson et. al. (Solar Energy, 84(9), 1717-1744) demonstrated, AM 1 solar spectrum better represents solar irradiance during summer noons in most places, when reflectance plays the most important role.

- Emittances are calculated for a temperature of 30 degrees Celsius to represent warm summertime weather.

- Care should be taken to distinguish between directional (near-normal) and hemispherical emittance. Most papers claim the directional value as the "emittance", but that is incorrect, and overestimates the true hemispherical emittance and thus the radiative cooling performance under open skies.

- Some of the data from the author's own works are improved versions of previously published works.

- Some of the data from older works (e.g. Polyvinylidene Chloride by Trombe and Grenier) correspond to reproductions by the author.

\section{Acknowledgements:}

The author would like to acknowledge Prof. Aaswath Raman of UCLA, and Prof. Yuan Yang and Prof. Nanfang Yu of Columbia University, for providing the optical and research tools used for this work. This work was supported by Schmidt Science Fellows, in partnership with the Rhodes Trust. 
History of different radiative cooling designs

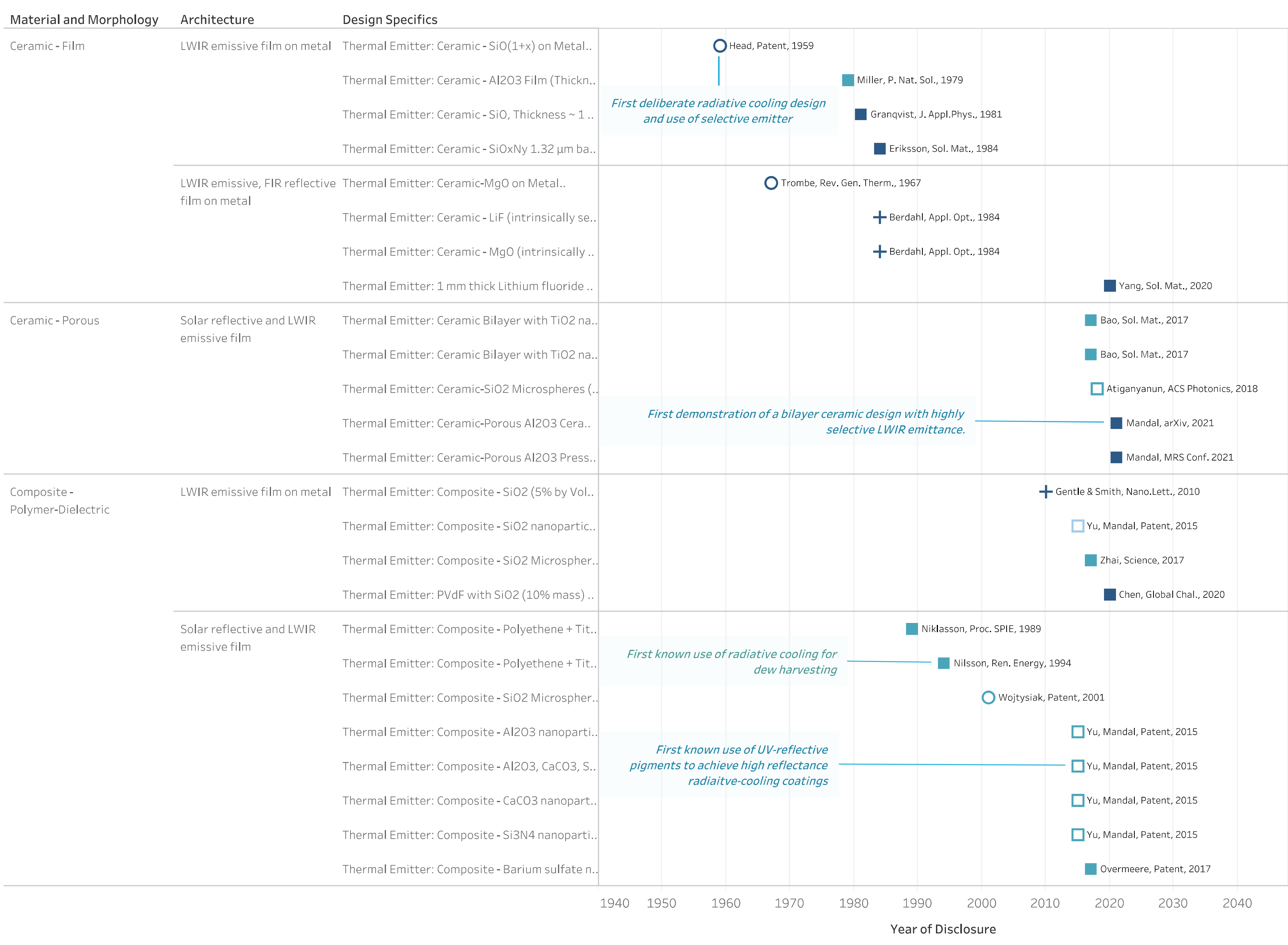

Demonstration

O conception

Experimental

( Experimental/Simulation (see notes)

+ Simulation

Emittance Type

Broadband

Broadband/Selective

Reflective

Selective

Semitransmissive 
History of different radiative cooling designs

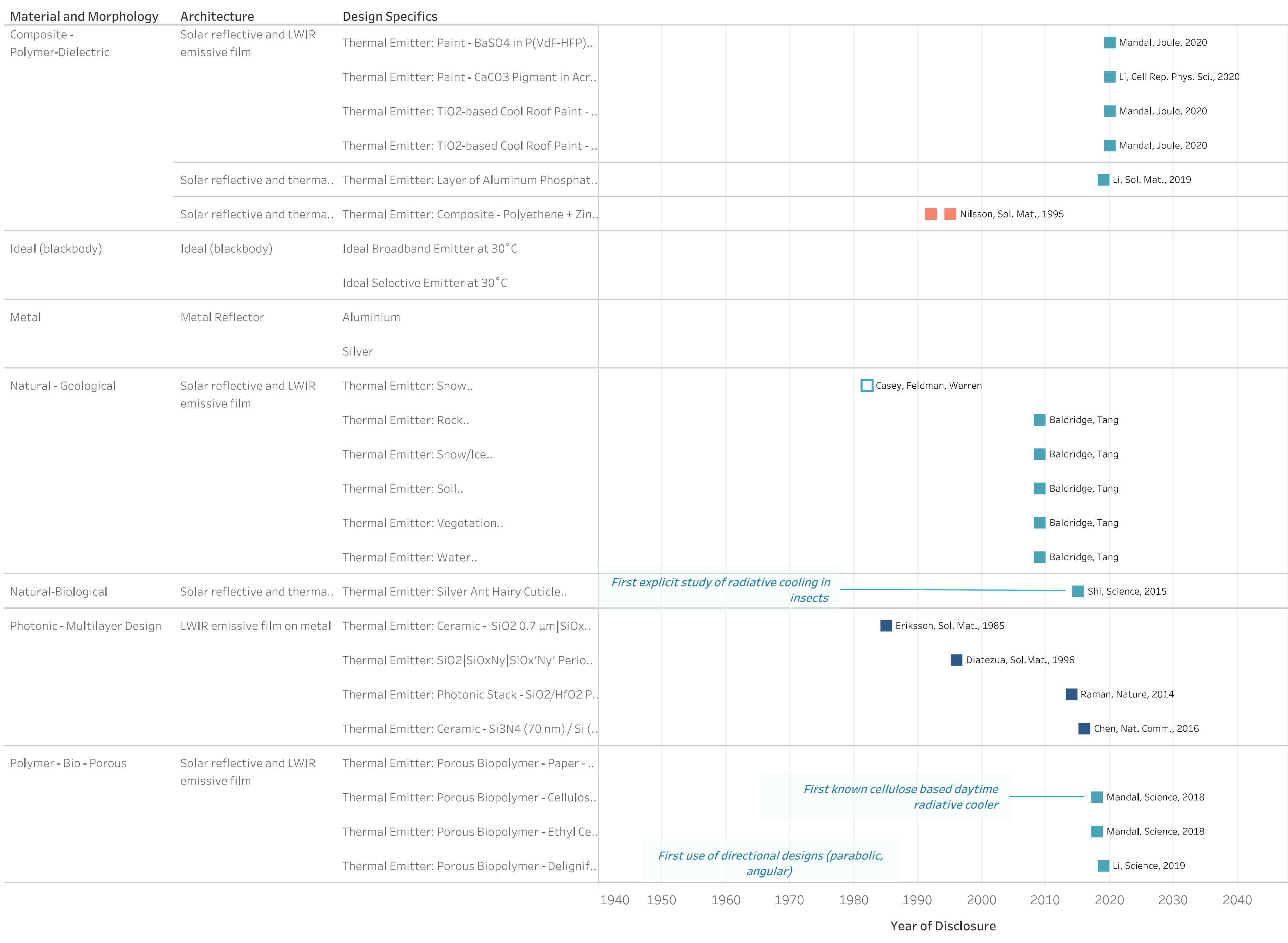

Demonstration

O conception

Experimental

․ Experimental/Simulation (see notes)

+ Simulation

Emittance Type

Broadband

Broadband/Selective

Reflective

Selective

Semitransmissive shows details about "Demonstration". Note: this list is not exhaustive, so it can only be used to say that a design has similar predecents, and NOT for claims of novelty. 
History of different radiative cooling designs

\begin{tabular}{lll} 
Material and Morphology & Architecture & Design Specifics \\
\hline Polymer - Blend - Film & LWIR emissive film on metal & Thermal Emitter: Polymer Blend - PDMS, PMI
\end{tabular}

Polymer - Film LWIR emissive film on metal Thermal Emitter: Polymer - Poly(vinyl chloride. Thermal Emitter: Polymer - Poly(4-methyl-1-P. Thermal Emitter: Polymer - PDMS, PMMA, PAA. Thermal Emitter: Polymer - PDMS film (thickn.. Thermal Emitter: Polymer - Poly(dimethyl silo. Thermal Emitter: Polymer - 3M Scotch Long La. Thermal Emitter: Polymer - PDMS film (thickn.. \begin{tabular}{lll}
\hline Polymer - Multilayer & Solar reflective and therma.. & Thermal Emitter: Polymer 3M ESR (multilayer . \\
\hline Polymer - Porous & Solar reflective and LWIR & Thermal Emitter: Porous Polymer - P(VdF-HFP).
\end{tabular} emissive film

Thermal Emitter: Porous Polymer - expanded P.

Thermal Emitter: Porous Polymer - Spectralon.

Thermal Emitter: Porous Polymer - P(VdF-HFP). Thermal Emitter: Porous Polymer - Sintered $\mathrm{P}$. Solar reflective and thermally emissive film, supplemented by metal refl.. Thermal Emitter: Porous Polymer - Poly(ethen. Solar reflective and thermally transmissive film

Thermal Emitter: Porous Polymer - Polyaceton. Thermal Emitter: Porous Polymer - Polyethene. Thermal Emitter: Porous Polymer - Polyethene. Thermal Emitter: Porous Polymer - Polyethene. 19401950

$\mid \begin{gathered}\text { Genier, Rev. Phys. Appl., 1979 } \\ \text { (reproduced with silver) }\end{gathered}$

First formal elucidation or radiative cooling

First demonstration of scalable polymeric radiative cooling film

First demonstration of substrate-independent paintable coatings with superhigh solar reflectance

Flrst waterborne radiative cooling paint with high solar reflectance

\begin{tabular}{|l|l|l|}
\hline Grinivasan, Appl. Phys. Lett., 2016 \\
\hline
\end{tabular}

Коu, ACS Phot., 2017

Mandal, arXiv, 2020 Song, Appl. Phys. Lett., 2017 Gentle \& Smith, Adv.Sci, 2015

\section{Mandal, Science, 2018}

Mandal, Joule, 2019

Mandal, Joule, 2020 Huang, Adv. Func. Mat., 2021 Yang, Solar Energy, 2018

[i, Nat.Nano, 2021



Demonstration O Conception

- Experimental

D Experimental/Simulation (see notes)

+ Simulation

Emittance Type

Broadband

Broadband/Selective

Reflective

Selective

Semitransmissive

Timeline of radiative cooling innovations, showing "Year of Disclosure" for each "Design Specifics", broken down by "Material and Morphology" and "Architecture". Color shows details about "Emittance Type". Shape shows details about "Demonstration". Note: this list is not exhaustive, so it can only be used to say that a design has similar predecents, and NOT for claims of novelty. 
Performances of Radiative Coolers - R Solar vs Directional $\varepsilon$ LWIR

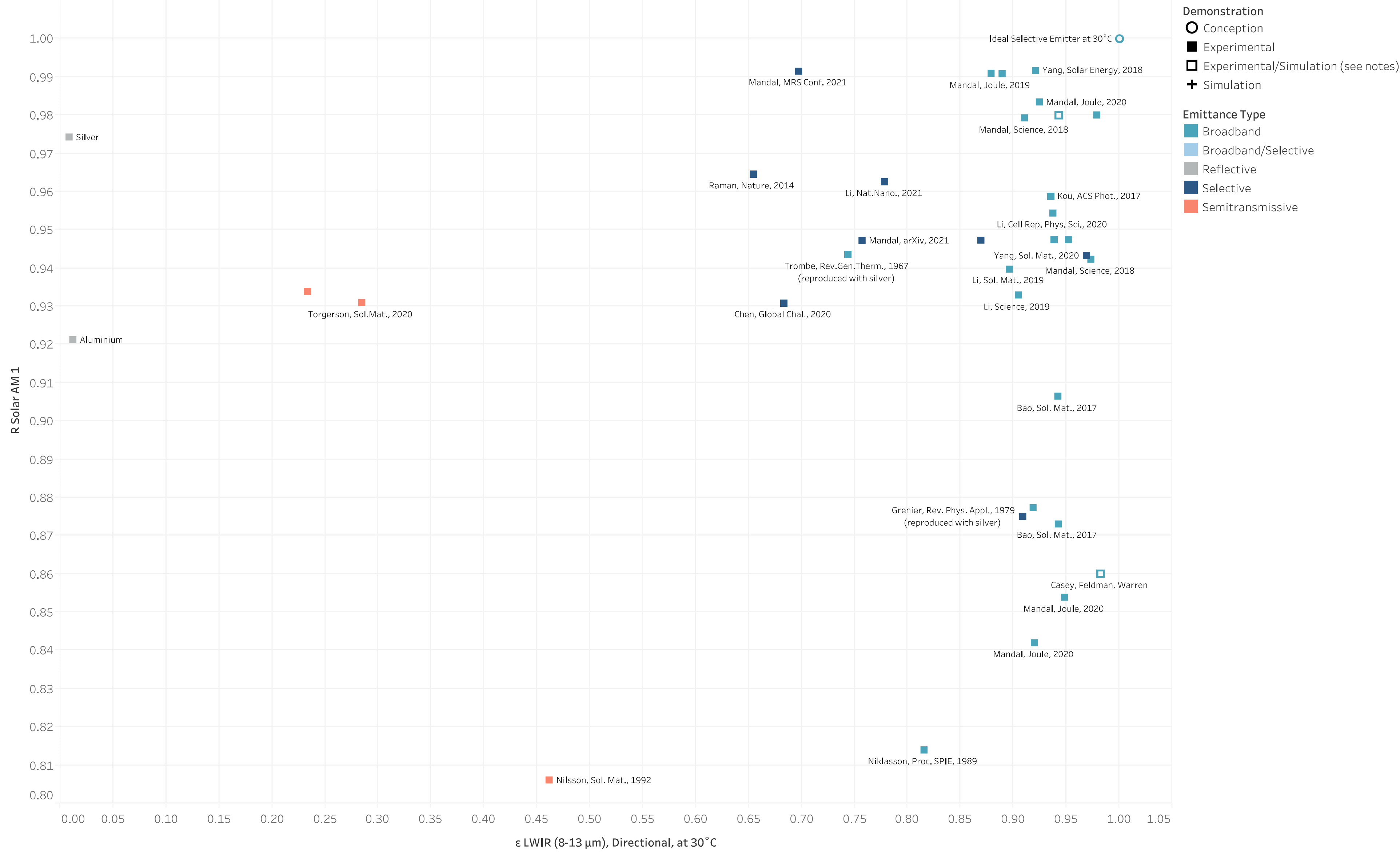

A survey of radiative coolers in the literature, showing their solar reflectances for a vertical incidence (R Solar AM 1), derived using the Air Mass 1 (AM 1 ) solar spectrum, and LWIR directional emittances ( $\varepsilon$ LWIR (8-13 $\mu \mathrm{m})$, Directional, at $30^{\circ} \mathrm{C}$ ), which are near-normal, unless mentioned otherwise in the notes. 
Performances of Radiative Coolers - $\varepsilon$ LWIR, Directional vs Hemispherical

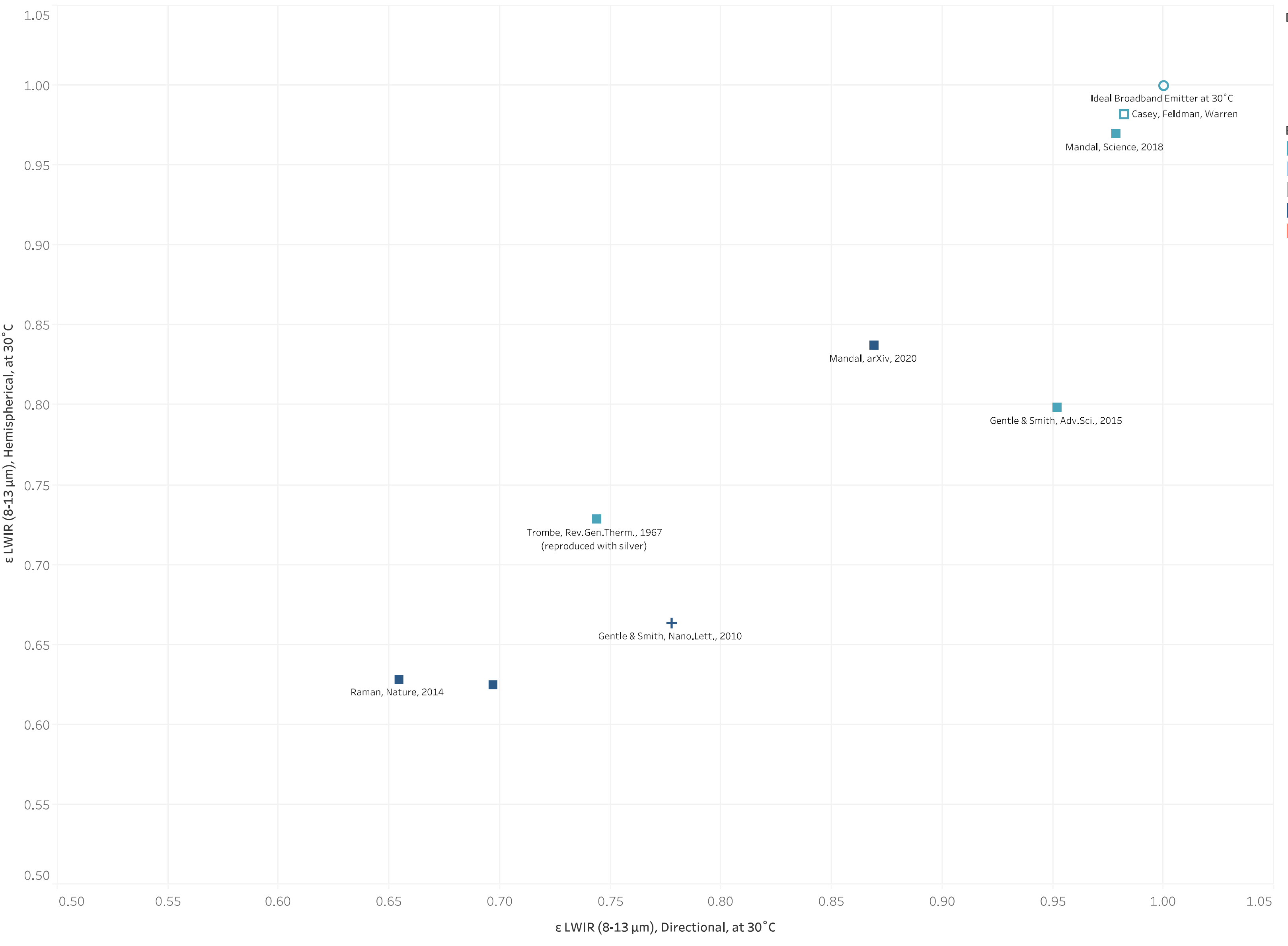

Demonstration

O conception

Experimental

ㅁperimental/Simulation (see notes)

+ Simulation

Emittance Type

Broadband

Broadband/Selective

Reflective

Selective

Semitransmissive

A survey of radiative coolers in the literature, showing their solar reflectances for a vertical incidence (R Solar AM 1 ), derived using the Air Mass 1 (AM 1 ) solar spectrum, and LWIR directional emittances ( $\varepsilon$ LWIR (8-13 $\mu \mathrm{m})$, Directional, at $30^{\circ} \mathrm{C}$ ), which are near-normal, unless mentioned otherwise in the notes. 